\title{
Bayesian Belief Networks for fault detection and diagnostics of a three- phase separator
}

\author{
M. Vileniskis, R. Remenyte-Prescott, D. Rama \& J. D. Andrews \\ Centre for Risk and Reliability Engineering, University of Nottingham, Nottingham, United Kingdom
}

\begin{abstract}
A three-phase separator (TPS) is one of the key components of offshore oil processing facilities. Oil is separated from gas, water and solid impurities by the TPS before it can be further processed. Failures of the TPS can lead to unplanned shutdowns and reduction of the efficiency of the whole oil processing facility as well as posing hazards to safety of personnel. A novel fault detection and diagnostic (FDD) methodology for the TPS is proposed in this paper. The core of the methodology is based on Bayesian Belief Networks (BBN). A BBN model is built to replicate the operation of the TPS: when the system is fault free or operating with single or multiple failed components. Results of the capabilities of the BBN model to detect and diagnose single and multiple faults of the TPS components are reported in this paper.
\end{abstract}

\section{INTRODUCTION}

The demand for oil products throughout the world requires the oil and gas processing facilities to work at maximum efficiency. For example, according to the U.S. Energy Information Administration approximately 93 million barrels of oil products were produced every day around the world in 2014 (U.S. Energy Information Administration, 2014). Thus, the unexpected shutdowns of offshore oil and gas plants due to equipment failures result in big economic losses.

One of such equipment is a three-phase separator (TPS), where a failure can cause the whole oil processing plant to be stopped. TPSs are used to separate water, gas and solid impurities from oil. Faults in TPS are commonly detected by using either thresholds of the process variables (Chan, 2005) (e.g. oil level, water level and etc.), statistical analysis of the process variables (Roverso, 2002, Omana \& Taylor, 2007, Taylor \& Omana, 2008) or precise mathematical models (Dias et al., 1993, Kinnaert et al., 2000, Al-Hajri \& Rossiter, 2010), which simulate the operation of the TPS and then compare its outputs with the readings of the actual TPS.

The first commonly used approach usually detects failures when their effect is already critical to the system and the shutting down of the TPS is unavoidable. The second approach needs historical data, which might not be available, especially for hazardous failure modes, since little data is available due to their rare occurrence. Finally, for the third approach, a very good understanding of process conditions is necessary and usually the model requires extensive changes, if operating conditions were due to change.

A fault detection and diagnostic (FDD) methodology based on Bayesian Belief Network (BBN) technique for TPS is proposed in this paper. The BBNs are used for modelling systems usually consisting of a number of different components and the complexity and uncertainty lies in the interactions between those components. For example aeroplane engines (Sahin et al., 2007) or water tank system (Lampis \& Andrews, 2009).

The BBN model was chosen due to several reasons, including graphical representation of the modelled system, inclusion of expert knowledge about failure modes of the system, ability to model uncertainties in a probabilistic way, ability to build the model in a structured and modular way, and update the prior knowledge about occurrence of certain failure modes without altering the structure of the model. The proposed methodology can cope with multiple faults, takes into account the condition of the components throughout time and exploits the combinations of sensor readings in order to derive supplementary information about the processes in the TPS without the need to install additional sensors.

This paper reports on the development of the methodology (section 3) and its application to a TPS simulation model (section 4), when single and multiple faults are introduced. The BBN technique is briefly introduced next. 


\subsection{Bayesian Belief Networks}

Bayesian Belief Network (BBN) is a causal network that has a quantitative representation of causal links within the network (Jensen \& Nielsen, 2007). A $\mathrm{BBN}$ consists of the following elements:

- A set of variables with a finite set of mutually exclusive states.

- A set of directed edges between the variables, which have to form an acyclic directed graph.

- Conditional probability tables (CPT), which express the causal relationships between the variables in a quantitative way.

BBNs can be built in a modular way, for example, in order to model the sections of the system that have an identical structure the same instance of BBN can be reused. BBNs that are built in a modular way, as in this study, are referred to as Object Oriented Bayesian Networks (OOBN) (Koller \& Pfeffer, 1997).

It is common to represent the BBN graphically. The variables of BBN are depicted by elliptical nodes joined together by arrows. A specific graphical notation that is used throughout this paper is given in Table 1. Note, that "Information node" and "Condition node" have different shadings in order to visually identify them in the $\mathrm{BBN}$ and is a nonstandard graphical representation. The basic principles of TPS operation, as well as the components of TPS, are presented in next section.

Table 1. Graphical notation used in representing BBNs

Node Name and purpose
marking
An "Instance node" is used to create a modular BBN.
If certain parts of the BBN are repetitive these can be
constructed as instances and then used throughout the
BBN.
An "Input node" is used to connect "Instance node"
to other nodes in the BBN. Input nodes must be cho-
sen when a BBN, which is used as an instance node,
is built.
An "Output node" is used to provide an output (or
multiple outputs) from the "Instance node".
An "Intermediate node" is used for intermediate cal-
culations and is neither input nor output nodes.
An "Information node" is used to provide evidence in
the BBN from sensor readings of the TPS. These
nodes are coloured in pale green.
A "Condition node" represents states of every com-
ponent of TPS (e.g. LT1 state, LC1 state) in the BBN
and is used to monitor the state of the TPS given sen-
sor readings. These nodes are coloured in blue.

\section{THREE-PHASE SEPARATOR}

Three-phase separator (TPS) is an essential equipment in the oil production and processing plant. The schematic of the TPS (Arnold \& Stewart, 2008) is given in Figure 1.

The TPS is used to separate gas and water from oil. The operational principle of the TPS is based on the laws of gravity: higher density material (e.g. water) settles on the bottom of the TPS, while lower density material (e.g. gas and oil) flow to the top of TPS. A horizontal TPS with a weir is considered in this study, as it is a commonly used TPS due to good separation quality, low initial set-up costs and ease of maintenance.

The TPS can be roughly split into several sections:

- A liquid separation section, where the separation of water and oil occurs (the section to the left of the weir).

- A separated oil section, where the separated oil flows over the weir from the liquid separation section (the section to the right of the weir).

- A separated gas section, where the remaining space from two previous sections is left for the gas phase.

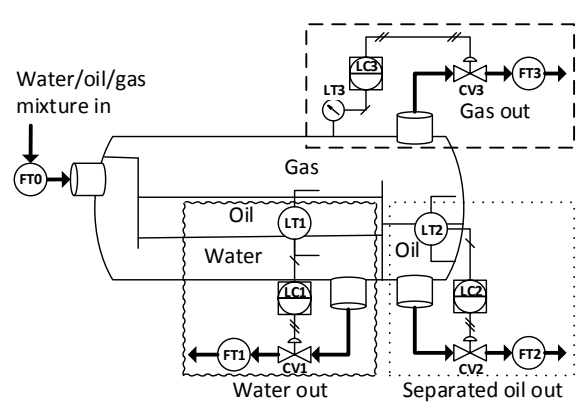

Figure 1. Horizontal three-phase separator schematic of configuration with a weir

The TPS is controlled by using PI controllers (Svrcek et al., 2006) to control the level of each phase (water level control loop is marked as a wavy rectangle, oil level control loop - as a dotted rectangle and pressure level control loop - as a dashed rectangle in Figure 1) in feedback control loops.

Failure of the components of the TPS could lead to improper control and thus lead to undesirable events, such as overpressure, under pressure, liquid carryover, gas blowby (American Petroleum Institute, 2001). Alarms are set to be triggered for the oil, water and pressure when the levels are too high or too low in the TPS in order to prevent the undesirable events.

\subsection{Simulation model of a three-phase separator}

In order to demonstrate the proposed methodology a simulation model of the TPS is built in this study. The system models can capture the main operating conditions of real systems and provide several benefits, when testing the FDD techniques (Dias et al., 1993, Omana \& Taylor, 2007):

- Ease of modelling different failures, e.g. even hazardous failure modes.

- Data is easily obtainable and the testing can be performed on a large number of different scenarios. 
- Low costs of implementing a simulation model, when compared to a scaled version of a real operating system.

Software with a graphical user interface to model the operation of the TPS was written as a part of this study in order to obtain sensor readings from the TPS, while operating under normal operating conditions and affected by failures.

Selected failure modes are considered for the components of the TPS. They are summarised in Table 2. The TPS is simulated until a predefined simulation time runs out, an alarm is raised or an undesirable event occurs. Further details on the simulation model can be found in (Vileiniskis et al., 2016). The proposed methodology is presented next.

Table 2. Failure modes of components of a three-phase separator

\begin{tabular}{ll}
\hline Component name & Failure mode \\
\hline Flow transmitter (FT0, FT1, FT2, & $1-$ Failed stuck (FS) \\
FT3) & \\
Level transmitter (LT1, LT2, LT3) & $1-$ Failed stuck (FS) \\
PI controller (LC1, LC2, LC3) & $1-$ Failed low (FL) \\
& $2-$ Failed high (FH) \\
Control valve (CV1, CV2, CV3) & $1-$ Failed closed (FC) \\
& $2-$ Failed opened (FO) \\
\hline
\end{tabular}

\section{PROPOSED METHODOLOGY}

Fault detection and diagnostics methodology based on Bayesian Belief Networks is proposed in this paper. The BBN model is developed in a modular way in order to replicate the undergoing processes in the TPS in each of the individual sections (an individual BBN is built for each section) before three separated liquids/gases, i.e. water, oil and gas, leave the vessel. Thus the final BBN is an OOBN model, as described in section 1.1.

The operation of the TPS is split into consecutive time intervals (called time segments in this study) and the sensor readings obtained from the TPS during these time intervals are fed into the OOBN. Analysis of the sensor readings with the OOBN is then performed and prediction on the most likely states of the components is made.

FDD capabilities of the proposed methodology are tested by modelling failures in the TPS simulation model and then using the outputs from the simulation model as evidence in the BBN model information nodes to obtain posterior probabilities of component failures in the corresponding condition nodes. An increase in the posterior probability of the failure mode of a particular component in the BBN will be considered as an indication of the presence of the failure of that component in the simulation model.

\subsection{Fusion of sensor readings}

One novelty of the proposed methodology is to combine the information from several sensors. In this way, supplementary data, such as changes of liquid/gas levels can be derived without a need to install additional sensors in the separator. The following combinations of sensors are obtained:

- FTO water - FT1. Determines the water level change rate in the separation section and aids the FDD of transmitters FT0, FT1 and LT1.

- FTO water - FT1 + FTO oil. Determines the total liquid level change rate in the separation section and aids the FDD of transmitters FT0, FT1 and LT1.

- FTO gas - FT3. Determines the gas change rate in the separator, which is used to identify pressure level change rate and aids the FDD of transmitters FT0, FT3 and LT3.

- FTO water - FT1 + FTO oil - FT2. Determines the volume change for gas in the separator and aids the FDD of FT0, FT1, FT2, LT1 and LT2.

- LT1 + LT2 level changes. Determines the volume change for gas in the separator and aids the FDD of transmitters FT0, FT1, FT2, LT1 and LT2.

For example, the combination "FT0 water - FT1" provides information on the water level change in the liquid separation section, which is also given by level transmitter LT1. This way, the sensor readings can be cross-checked to identify any discrepancies, which could indicate a possible failure of one of the components.

\subsection{BBN for a control loop}

A generic BBN for a control loop is given in Figure 2.

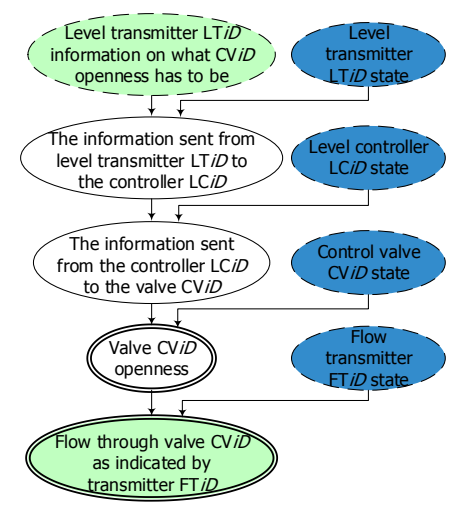

Figure 2. BBN for a control loop "FTiD given level LTiD"

It is built adhering to the logic that the information flow would follow in a physical system:

1 Level transmitter $\mathrm{LT} i D$ reads the information about the level of one of the phases and depending, on the state of the transmitter, sends this information to the controller LCiD.

2 The controller LCiD in turn sends the corresponding command (based on the state of the controller) to the control valve $\mathrm{CV} i \mathrm{D}$. 
3 The valve CViD opens according to its state and the command it received from the controller LCiD.

4 Flow through valve $\mathrm{CViD}$ is indicated by a flow transmitter FTiD, which indicates the value based on the opening of the CViD and the state of the flow transmitter.

\subsection{BBN for the liquid separation section}

The BBN for the liquid separation section (see Figure 3) has an instance of the previously developed BBN "FTiD given level LTiD" (Figure 2), where it is used to represent the water control loop.

The liquid separation section contains two phases (water and oil) flowing into the section and two phases flowing out of the section (separated oil is flowing over the weir to a separated oil section and separated water is leaving the TPS through valve CV1). The weir limits the total liquid amount in the section as well as the amount of the separated oil that is flowing into the next section. The BBN for this section is developed by considering the process of the liquid propagation through this section, as well as possible changes of liquid levels.

An algorithm was developed to derive the CPTs for the nodes in this BBN and it is based on the arithmetic operations that have to be performed on the parent node information. For example, the CPT for the node "FT0 water - FT1" was derived by looping through possible water inflow and outflow values and calculating the difference between them. The information shown by the combination of transmitters then depends on the condition of both transmitters FT0 and FT1. For full details of the algorithm refer to (Vileiniskis et al., 2016).

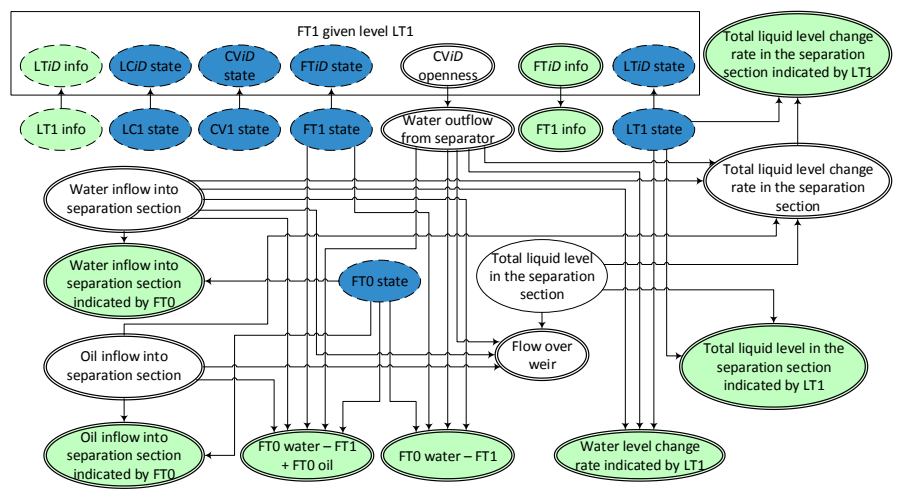

Figure 3. BBN for the liquid separation section

\subsection{BBN for the separated oil section}

The BBN built for the separated oil section is given in Figure 4.

An instance of the control loop BBN "FT2 given level LT2" is used to model the control of oil level. The level of oil in the separated oil section depends on the amount of oil flowing from the liquid separation section (node "Oil inflow from the separation section") and the amount of oil leaving the TPS through valve CV2 (node "Oil outflow from separator"). The change of oil level is observed through readings of transmitter LT2 (node "Oil level change rate in the separated oil section indicated by LT2").

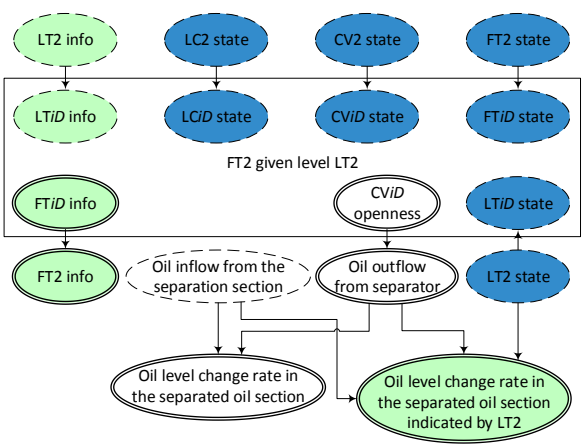

Figure 4. BBN for the separated oil section

\subsection{BBN for the separated gas section}

The BBN for the separated gas section is given in Figure 5. An instance of BBN for the control loop "FT3 given level LT3" is used to model the control of pressure level. This section is only considered to have gas flowing into the section and then gas leaving the TPS through valve CV3. Differently from other phases (oil and water), gas is compressible. Thus the pressure level inside the TPS depends on the gas amount, as well as the volume that is available for gas (nodes "Gas level change rate" and "Change of volume for gas in the separator" are made parents of a node "Pressure level change rate").

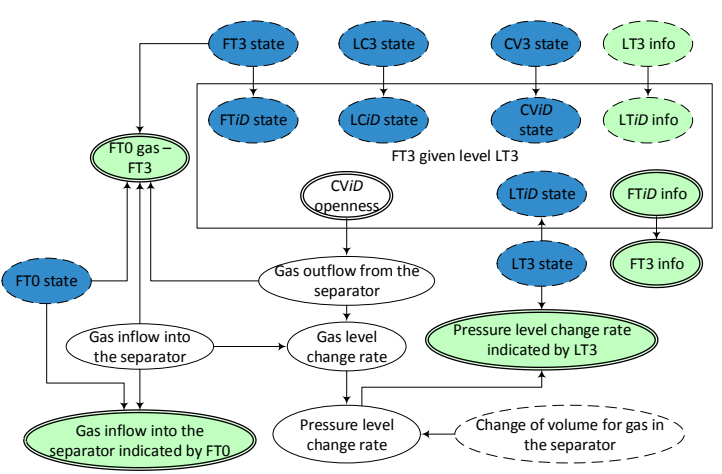

Figure $5 . \mathrm{BBN}$ for the separated gas section

\subsection{BBN for the interactions between individual sections}

The BBN for the interactions between the individual sections, given in Figure 6, contains an instance of each individual section BBN (previously described in sections 3.3, 3.4 and 3.5). The individual sections are connected in the following way:

- "Liquid separation section" is connected to "Separated oil section" by making the node "Flow over weir" as a parent node of a node "Oil inflow from the separation section".

- "Liquid separation section" and "Separated oil section" are connected to "Separated gas section" 
by making the nodes "Water inflow into separation section", "Water outflow from separator", "Oil inflow into separation section" and "Oil out- flow from separator" as parent nodes of a node "Change of volume for gas in the separator".

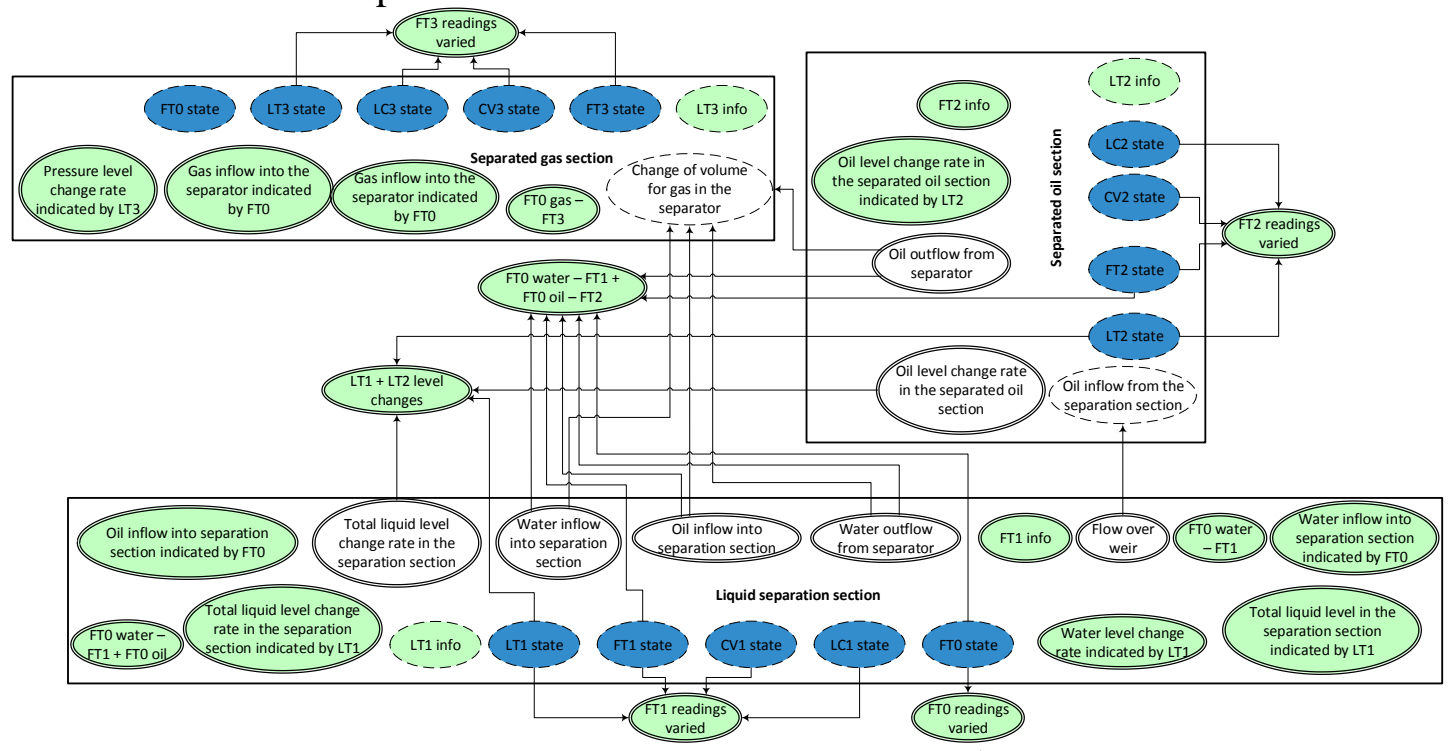

Figure 6. BBN for the interactions between individual sections "Single time segment"

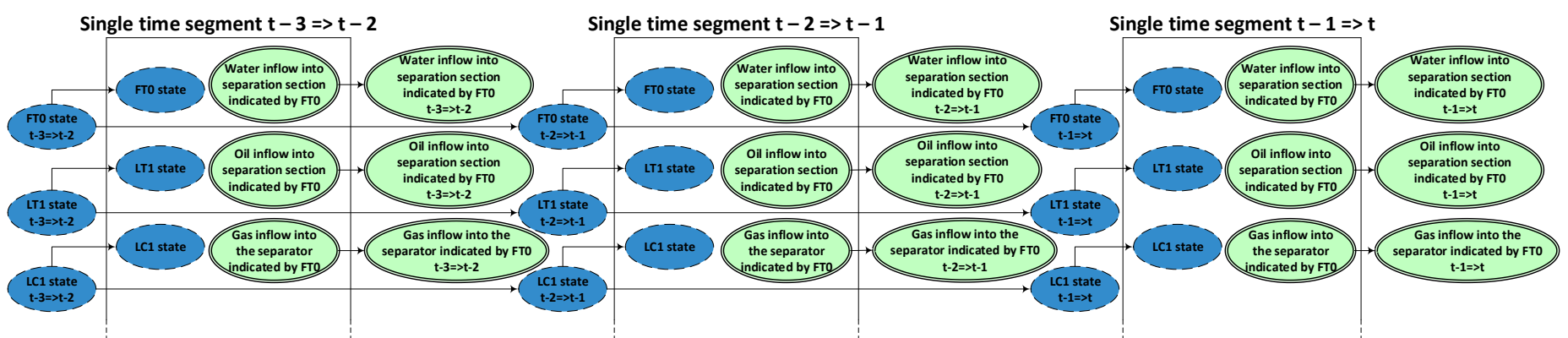

Figure 7. Excerpt from a triple time segment OOBN for fault detection and diagnostics

Two information nodes ("LT1 + LT2 level changes" and "FT0 water - FT1 + FT0 oil - FT2") are used to input the information on how the volume available for gas changes in the TPS. The developed BBN is called "Single time segment" in this study, since it uses the sensor readings obtained from the TPS for single time segment, as explained at the beginning section 3 .

\subsection{Triple time segment $B B N$ for fault detection and diagnostics}

A triple time segment OOBN (see an excerpt in Figure 7) is created by joining three instances of the "Single time segment" BBNs, given in Figure 6, to perform FDD of the TPS. The instances of "Single time segment" BBNs are labelled with suffixes " $t$ $3=>t-2$ ", " $t-2=>t-1$ " and " $t-1=>t$ " to indicate the time segment of the sensor readings that are used in the $\mathrm{BBN}$. For example, if a time segment is 10 seconds long, then the " $t-3=>t-2$ " means that the BBN uses the sensor readings from a time segment of 30 to 20 seconds from the most recent readings.

This OOBN is then used for the FDD of the TPS. The procedure can be summarised in the following steps:

1 Enter the sensor readings into the OOBN.
2 Update the posterior probabilities throughout the OOBN, given the new sensor readings.

3 Track how the posterior probabilities of the condition nodes in the most recent time segment changes (nodes with a suffix " $t-1=>t$ ") in order to detect and identify failures of components (a threshold of posterior probabilities for FDD has to be set (Threshold 1)).

4 Check if the posterior probabilities of the condition nodes significantly differ from the prior probabilities of the same condition nodes in adjacent time segments (a threshold is used to ignore very small differences between the prior and posterior probabilities).

5 If the difference between posterior and prior probabilities found in step 4 is significant, make posterior probabilities of the condition nodes as prior probabilities of the condition nodes in a previous time segment.

6 Repeat the procedure described in steps 1 to 5 when new sensor readings become available.

Such an approach allows conditions of components of the TPS to be monitored once sensor readings become available for all three time segments following the initial start of the monitoring.

For a more detailed description of each step as well as the details of CPTs quantification and the 
rules to build the section BBNs, refer to (Vileiniskis et al., 2016).

\section{APPLICATION OF THE METHODOLOGY}

The methodology is applied to a simulation model of the TPS, when single and multiple faults are present in the TPS. The testing scenarios for both single and multiple faults are summarised in Table 3 . Note that Threshold 1 and Threshold 2 in Table 3 are the thresholds necessary for FDD, as explained in section 3.7 (steps 3 and 4, respectively).

Table 3. Design of scenarios to test the FDD of OOBN with single and multiple faults

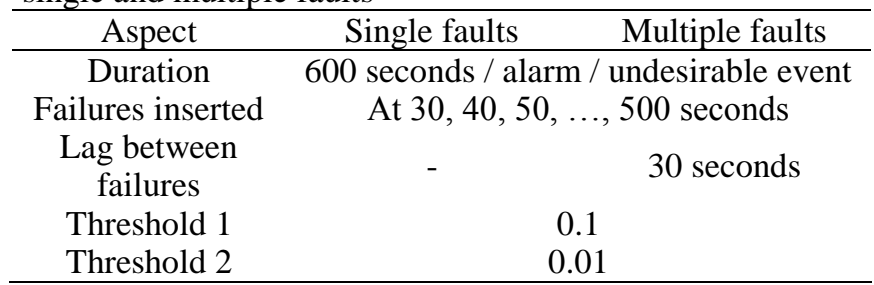

For each correctly detected and diagnosed failure mode its detection and diagnostics potential is determined using the following equation

$$
R_{i}=\frac{T_{i}}{T_{i}^{p}} \times 100 \%,
$$

where $R_{i}$ is the ratio of FDD capability of a specific failure mode $i, T_{i}^{d}$ is the time duration, when the $\mathrm{BBN}$ detected the presence of a failure mode $i, T_{i}^{p}$ is the time duration the failure mode $i$ was present in the simulation model.

Similarly, the measure of false alarms is given as a ratio expressed using the following equation:

$$
R_{i F}=\frac{T_{i F}}{T_{t}} \times 100 \%,
$$

where $R_{i F}$ is the ratio of false alarm of a failure mode $i, T_{i F}$ is the time duration, when the BBN falsely detected the presence of a failure mode $i, T_{t}$ is the total time of simulation.

The notation for the indices of ratios used in equations (1) and (2) is given in Table 4. The capabilities of BBN for FDD of single faults are considered first.

Table 4. Notation of failure modes

\begin{tabular}{llll}
\hline Failure mode & Index $i / i F$ & Failure mode & Index $i / i F$ \\
\hline FT0 FS & $1 / 1 \mathrm{~F}$ & CV2 FC & $11 / 11 \mathrm{~F}$ \\
LT1 FS & $2 / 2 \mathrm{~F}$ & CV2 FO & $12 / 12 \mathrm{~F}$ \\
LC1 FH & $3 \mathrm{~F}$ & FT2 FS & $13 / 13 \mathrm{~F}$ \\
LC1 FL & $4 \mathrm{~F}$ & LT3 FS & $14 / 14 \mathrm{~F}$ \\
CV1 FC & $5 / 5 \mathrm{~F}$ & LC3 FH & $15 \mathrm{~F}$ \\
CV1 FO & $6 / 6 \mathrm{~F}$ & LC3 FL & $16 \mathrm{~F}$ \\
FT1 FS & $7 / 7 \mathrm{~F}$ & CV3 FC & $17 / 17 \mathrm{~F}$ \\
LT2 FS & $8 / 8 \mathrm{~F}$ & CV3 FO & $18 / 18 \mathrm{~F}$ \\
LC2 FH & $9 \mathrm{~F}$ & FT3 FS & $19 / 19 \mathrm{~F}$ \\
LC2 FL & $10 \mathrm{~F}$ & & \\
\hline
\end{tabular}

\subsection{Fault detection and diagnostics of single faults}

All the single failure modes (except for the controller faults (LC1 FL/FH, LC2 FL/FH and LC3 $\mathrm{FL} / \mathrm{FH}$ ), since they have the same effect as failures of the corresponding valve, and thus it is impossible to distinguish between them) were inserted at different time points during the execution of the simulation in order to calculate the duration of detection lags of the faults. The results are summarised in Figure 8 .

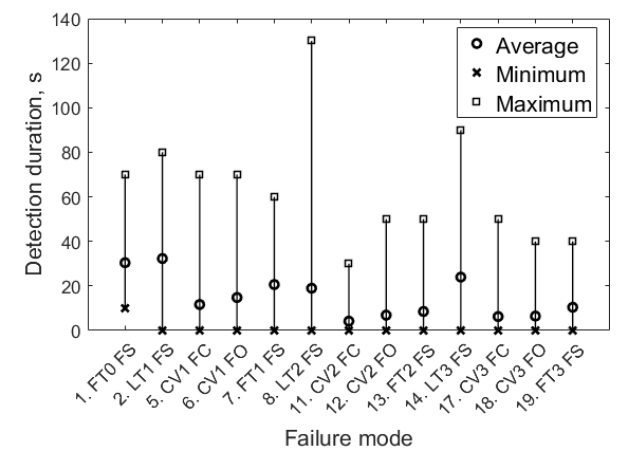

Figure 8. Fault detection duration for different failure modes, when single faults were inserted in the simulation model

As one can observe from Figure 8, the average fault detection durations were no longer than $40 \mathrm{sec}-$ onds, which should give enough time for the operator to perform actions to prevent any undesirable event. The maximum fault detection duration was observed for the failure mode level transmitter LT2 being failed stuck. In one case, this failure mode was only detected by the BBN model 130 seconds after it was inserted at 220 seconds into the simulation model. Even though the detection of the failure in this case took significantly longer than the average time (18.96 seconds), the failure was still detected approximately 60 seconds before the separated oil level reached the level, where it would raise an alarm if the level transmitter LT2 was not failed. Thus the methodology still gives an earlier indication of a failure than that provided by the alarm methods.

Table 5. Summary of the FDD ratios, when a single fault has been inserted in the simulation model

\begin{tabular}{ccl}
\hline$i$ & \multicolumn{1}{c}{$R_{i}$} & \multicolumn{1}{c}{ Additional faults detected, $R_{i F}$} \\
\hline 1 & 76.43 & - \\
2 & 81.79 & \\
5 & 90.12 & $R_{4 F}=29.32$ \\
6 & 89.89 & $R_{3 F}=33.60, R_{19 F}=1.06, R_{15 F}=R_{18 F}=0.32$ \\
7 & 74.32 & $R_{3 F}=R_{6 F}=1.40, R_{4 F}=R_{5 F}=1.26$ \\
8 & 84.34 & $R_{2 F}=0.17$ \\
11 & 95.29 & $R_{10 F}=25.30$ \\
12 & 90.41 & $R_{9 F}=20.46$ \\
13 & 96.64 & $R_{10 F}=R_{11 F}=0.25, R_{9 F}=R_{12 F}=0.22$ \\
14 & 92.66 & \\
17 & 96.47 & $R_{16 F}=42.43$ \\
18 & 96.47 & $R_{15 F}=41.27$ \\
19 & 93.91 & $R_{15 F}=1.33, R_{17 F}=1.29, R_{16 F}=1.15, R_{16 F}=0.93$ \\
\hline
\end{tabular}

The biggest challenge for the BBN model was the detection of transmitter failures (for example $i=1$ 
for FT0 FS and $i=7$ for FT1 FS, where $R_{i}$ was lowest, in Table 5). This was due to the fact that the failure effect depends on the time of occurrence of the failure. In some cases the failure might be a hidden failure for a certain period of the simulation. However, the failure becomes hidden because of specific operating conditions and thus the performance of any of the techniques for FDD would suffer due to this phenomenon.

The ratios $R_{i F}$ are very low (not higher than 1.4), except for the false diagnostics of controller failures, such as $R_{4 F}=29.32$, when CV1 FC failure mode was inserted, but this occurred due to the nature of the controller failures, as explained at the beginning of section 4.1.

\subsection{Fault detection and diagnostics of multiple faults}

Two failure modes occurring in a single simulation run are modelled next. The multiple failures have been inserted in the simulation model in the following way: first one of the level transmitter failures (LT1 FS, LT2 FS or LT3 FS) is introduced and then it is followed by one of the remaining possible failures (except for controller failures). In total, 36 combinations of two failures were obtained giving a total of 1728 (failures are inserted at 48 time points) scenarios to test the capabilities of FDD with multiple faults. Note that, only the results for combinations with LT1 FS are presented in this section, as the same trends exist for failures LT2 FS and LT3 FS.

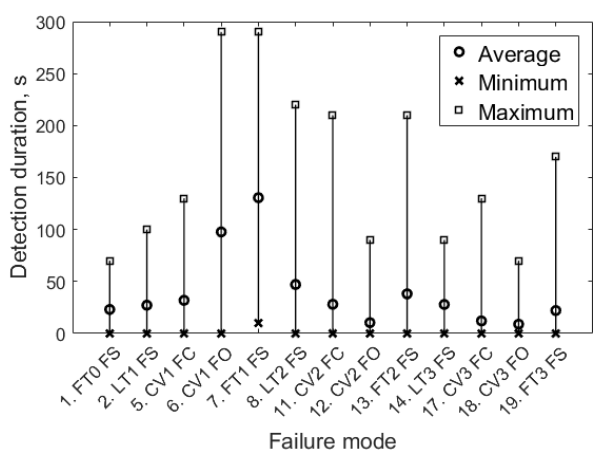

Figure 9. Fault detection duration for different failure modes, when multiple faults were inserted in simulation model

The average detection durations of multiple faults are given in Figure 9. Note that, only a single fault label is given in the plot to avoid repeating the label LT1 FS. The difference from Figure 8 is that for the LT1 FS failure mode, the detection duration is averaged through all of the 576 scenarios (since it was inserted together with all remaining 12 failure modes); whilst for other failure modes the duration is averaged through 48 scenarios.

Detection lags have remained similar for most of the failure modes if compared to the single faults scenarios (see Figure 8). However, for the failure modes CV1 FO and FT1 FS, the average detection times have increased quite significantly (from 14.79 to 97.71 seconds and from 20.62 to 130.62 seconds, respectively). When the individual scenarios with the longest detection durations were analysed in detail, the findings suggested that:

- Some of the failures are hidden failures due to the operating conditions of TPS at the time they occur.

- The effect of failure mode LT1 FS hides the effect of failures of components that are associated or dependant on LT1, e.g. valve CV1.

Similarly as for the single faults case, the results with multiple faults are summarised in Table 6 with FDD ratios as expressed by equations (1) and (2).

Table 6. Summary of the FDD ratios, when multiple faults have been inserted in the simulation model

\begin{tabular}{|c|c|c|c|}
\hline$i$ & $R_{i}$ & $R_{2}$ & Additional faults detected, $R_{i F}$ \\
\hline 1 & 68.03 & 73.33 & $\begin{array}{l}R_{7 F}=0.99, R_{10 F}=0.74, R_{13 F}=0.69, R_{l 6 F}= \\
R_{19 F}=0.49, R_{11 F}=0.39, R_{12 F}=0.35, R_{8 F}= \\
0.10, R_{9 F}=R_{15 F}=R_{18 F}=0.30, R_{l 7 F}=0.20\end{array}$ \\
\hline 5 & 72.08 & 82.37 & $\begin{array}{l}R_{4 F}=21.15, R_{1 O F}=R_{11 F}=R_{13 F}=1.87, R_{19 F}= \\
0.96, R_{16 F}=0.64, R_{15 F}=R_{17 F}=R_{18 F}=0.32\end{array}$ \\
\hline 6 & 51.95 & 88.48 & $\begin{array}{l}R_{3 F}=22.08, R_{19 F}=0.87, R_{8 F}=0.35 \\
R_{15 F}=R_{18 F}=0.26\end{array}$ \\
\hline 7 & 11.32 & 81.79 & $\begin{array}{l}R_{10 F}=R_{11 F}=R_{13 F}=0.64 \\
R_{3 F}=R_{4 F}=R_{5 F}=R_{6 F}=0.20\end{array}$ \\
\hline 8 & 62.50 & 81.49 & $\begin{array}{l}R_{19 F}=3.64, R_{13 F}=2.34, R_{10 F}=R_{l 6 F}=R_{I 7 F}= \\
2.14, R_{l 1 F}=2.09 R_{15 F}=R_{18 F}=1.29\end{array}$ \\
\hline 11 & 75.71 & 77.54 & $\begin{array}{l}R_{10 F}=22.66, R_{16 F}=R_{17 F}=R_{19 F}=1.86 \\
R_{9 F}=17.67, R_{18 F}=R_{19 F}=2.81, R_{15 F}=2.75\end{array}$ \\
\hline 12 & 77.46 & 68.34 & $\begin{array}{l}R_{13 F}=1.43, R_{10 F}=R_{11 F}=1.31 \\
R_{16 F}=R_{17 F}=0.24\end{array}$ \\
\hline 13 & 71.29 & 81.79 & $R_{1 O F}=14.36, R_{11 F}=14.21, R_{9 F}=R_{12 F}=0.10$ \\
\hline 14 & 76.38 & 81.79 & 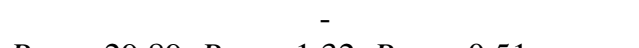 \\
\hline 17 & 86.91 & 80.65 & $\begin{array}{l}R_{16 F}=29.89, R_{19 F}=1.32, R_{18 F}=0.51 \\
R_{15 F}=0.10\end{array}$ \\
\hline 18 & 89.86 & 72.71 & $R_{15 F}=21.85, R_{10 F}=R_{11 F}=R_{13 F}=0.23$ \\
\hline 19 & 73.55 & 81.79 & $\begin{array}{l}R_{16 F}=7.05, R_{17 F}=6.07, R_{18 F}=5.08, R_{15 F}= \\
4.83, R_{8 F}=0.30, R_{7 F}=R_{14 F}=0.05\end{array}$ \\
\hline
\end{tabular}

This time, the BBN falsely identified a bigger amount of failure modes when compared to the single faults case (see Table 5). However, the ratios $R_{i F}$ are still reasonably low, except for the false diagnostics of controller failures. The BBN encountered problems, when detecting the failure FT1 FS ( $i=7$ in Table 6) together with LT1 FS. This was due to the fact that the flow transmitter FT1 always failed later than level transmitter LT1, thus the failure of FT1 FS had no effect on FT1 readings. This is just reconfirmed when looking at the numbers of undetected faults during the tested scenarios. For each combination of faults (LT1 FS and a second fault) a number of scenarios (out of 48), when either of the faults were undetected, are summarised in Table 7.

43 times out of 48 scenarios, the FT1 FS failure was undetected when inserted after failure mode LT1 FS. The failure of level transmitter LT1 has been detected in the majority of the scenarios ( 568 out of 576). In 7 out of 8 scenarios when LT1 was undetected, the effect of second failure was immedi- 
ate and the TPS simulation model ran only for a few time steps before an alarm was raised due to a too low/high separated oil level, and the simulation model was stopped before the failure of LT1 could be detected (average detection time for a single fault was 32.29 seconds), since the water level in the liquid separation section remained around set point as expected.

The BBN model has failed to detect the failures of components that are in the same control loop as the LT1 in a lot of scenarios, for example, FT1 FS (43 out of 48 ) or CV1 FO (24 out of 48 ). This was due to the hidden failures, when the second fault has no effect on the TPS, because of the first fault, as explained previously. However, note that there were no scenarios where both of the failures would be left undetected during the same simulation run, as they were undetected in separate simulation runs.

Table 7. Summary of undetected failures, when multiple faults (Fault 1 - LT1 FS) were inserted in the simulation model

\begin{tabular}{lcclcc}
\hline Fault 2 & Fault 1 & Fault 2 & Fault 2 & Fault 1 & Fault 2 \\
\hline FT0 FS & 1 & 0 & CV2 FO & 5 & 4 \\
CV1 FC & 0 & 19 & FT2 FS & 0 & 11 \\
CV1 FO & 0 & 24 & LT3 FS & 0 & 3 \\
FT1 FS & 0 & 43 & CV3 FC & 0 & 1 \\
LT2 FS & 0 & 9 & CV3 FO & 0 & 2 \\
CV2 FC & 2 & 7 & FT3 FS & 0 & 1 \\
\hline
\end{tabular}

\section{CONCLUSIONS AND FUTURE WORK}

In this paper, several novel aspects were proposed for the fault detection and diagnostics of a threephase separator. The proposed methodology is built in a modular way; it also takes into account the condition of components in previous time segments, exploits the combinations of transmitter readings and includes detection and diagnostics of multiple failure modes. FDD of multiple failure modes is a major improvement when compared to the previouslyproposed approaches.

One of the important features that could extend the proposed methodology is leak detection, as in some cases it might be a critical failure of the TPS. The proposed methodology is based on monitoring the changes of the liquid/gas in the three-phase separator using sensor readings and does not take into account the actual levels of liquid/gas (except when a PI controller output is calculated). The addition of actual levels of liquids/gas and their propagation from one time segment to another could be also included in the methodology. Another extension to the proposed methodology could be to include modelling other component failure modes, for example, valve being stuck half open/closed, and optimising the choice of thresholds for the FDD process.

\section{REFERENCES}

AL-HAJRI, E. M. \& ROSSITER, J. A. A unified frame work for oil producing stations using Petri nets. Control 2010, UKACC International Conference on, 7-10 Sept. 2010 2010. 1-8.

AMERICAN PETROLEUM INSTITUTE 2001. API RP 14C: Recommended Practice for Analysis, Design, Installation, and Testing of Basic Surface Safety Systems for Offshore Production Platforms. Protection concepts and safety analysis.

ARNOLD, K. \& STEWART, M. 2008. Chapter 5 - ThreePhase Oil and Water Separation. In: STEWART, K. A. (ed.) Surface Production Operations (Third Edition). Burlington: Gulf Professional Publishing.

CHAN, C. W. 2005. An expert decision support system for monitoring and diagnosis of petroleum production and separation processes. Expert Systems with Applications, 29, 131-143.

DIAS, A. C., BHAYA, A. \& KASZKUREWICZ, E. Fault Diagnosis in an Oil Production Plant Prototype Using a Diagnostic Model Processor. American Control Conference, 1993, 2-4 June 1993 1993. 107-111.

JENSEN, F. V. \& NIELSEN, T. D. 2007. Bayesian Networks and Decision Graphs, Springer.

KINNAERT, M., VRANČIĆ, D., DENOLIN, E., JURIČIĆ, Đ. \& PETROVČIĆ, J. 2000. Model-based fault detection and isolation for a gas-liquid separation unit. Control Engineering Practice, 8, 1273-1283.

KOLLER, D. \& PFEFFER, A. Object-oriented Bayesian networks. Proceedings of the Thirteenth conference on Uncertainty in artificial intelligence, 1997. Morgan Kaufmann Publishers Inc., 302-313.

LAMPIS, M. \& ANDREWS, J. D. 2009. Bayesian Belief Networks for System Fault Diagnostics. Quality and Reliability Engineering International, 25, 409-426.

OMANA, M. \& TAYLOR, J. H. Fault Detection and Isolation Using the Generalized Parity Vector Technique in the Absence of an a Priori Mathematical Model. Control Applications, 2007. CCA 2007. IEEE International Conference on, 1-3 Oct. 2007 2007. 970-975.

ROVERSO, D. 2002. Plant diagnostics by transient classification: The ALADDIN approach. International Journal of Intelligent Systems, 17, 767-790.

SAHIN, F., YAVUZ, M. C., ARNAVUT, Z. \& ULUYOL, O. 2007. Fault diagnosis for airplane engines using Bayesian networks and distributed particle swarm optimization. Parallel Computing, 33, 124-143.

SVRCEK, W. Y., MAHONEY, D. P. \& YOUNG, B. R. 2006. A Real-Time Approach to Process Control, John Wiley II\& Sons.

TAYLOR, J. H. \& OMANA, M. Fault Detection, Isolation and Accommodation Using the Generalized Parity Vector Technique. In: CHUNG, M. J. \& MISRA, P., eds. Proceedings of the 17th IFAC World Congress, 2008, 2008 COEX, South Korea 1914-1921.

U.S. ENERGY INFORMATION ADMINISTRATION. 2014. International Energy Statistics [Online]. Available: http://www.eia.gov/cfapps/ipdbproject/iedindex3.cfm [Accessed 10/12/2015.

VILEINISKIS, M., REMENYTE-PRESCOTT, R., RAMA, D. \& ANDREWS, J. 2016. Fault detection and diagnostics of a three-phase separator. Journal of Loss Prevention in the Process Industries, 41, 215-230. 